\title{
Intoxicação experimental por Aspergillus clavatus em ovinos ${ }^{1}$
}

\author{
Pedro S. Bezerra Júnior ${ }^{2}$, Adriana da Silva Santos ${ }^{3}$, Paulo Mota Bandarra ${ }^{3}$, \\ Pedro M. Ocampos Pedroso ${ }^{3}$, Saulo Petinatti Pavarini ${ }^{3}$, Andréia Spanamberg ${ }^{4}$, \\ Laerte Ferreiro ${ }^{4}$ e David Driemeier ${ }^{3^{*}}$
}

\begin{abstract}
Bezerra Jr P.S., Santos A.S., Bandarra P.M., Pedroso P.M.O., Pavarini, S.P., Spanamberg A., Ferreiro L. \& Driemeier D. 2009. [Experimental poisoning by Aspergillus clavatus in sheep.] Intoxicação experimental por Aspergillus clavatus em ovinos. Pesquisa Veterinária Brasileira 29(3):205-210. Setor de Patologia Veterinária, Universidade Federal do Rio Grande do Sul, Av. Bento Gonçalves 9090, Porto Alegre, RS 91540-000, Brazil. E-mail: davetpat@ufrgs.br

This paper describes the experimental reproduction of a neurological condition in sheep by the administration of a beer by-product contaminated with Aspergillus clavatus. Samples of this by-product, in which pure cultures of $A$. clavatus grew, originated from two farms where outbreaks of $A$. clavatus poisoning in cattle had occurred. The onset of symptomatology was 2 to 6 days after dosage with the contaminated beer by-product or pure A. clavatus culture. The clinical course lasted from one and a half to 12 days. Clinical signs were predominantly of locomotor and respiratory nature and included muscle tremors, hyperesthesia, and progressive tachypnea, rigidity of the pelvic limbs, posterior weakness, and recumbency. One sheep also showed occasional knuckling of fetlocks of the hind limbs. Gait abnormalities and tremors were more pronounced after exercise. In 6 of 7 sheep, appetite and dypsia were maintained until close to death or euthanasia. The main histological findings consisted of chromatolytic neuronal degeneration and necrosis in selected nuclei of the brain stem, the ventral horn of the spinal cord and the spinal, trigeminal, stellate and celiac ganglions. Three sheep also presented slight degenerative and necrotic changes in muscles of the pelvic and thoracic limbs.
\end{abstract}

INDEX TERMS: Aspergillus clavatus, sheep, beer by-product, poisoning, neuronal chromatolysis.

RESUMO.- Descreve-se a reprodução experimental de doença neurológica em ovinos através da administração de bagaço de malte (resíduo cervejaria) contaminado com Aspergillus clavatus. Esse resíduo de cervejaria, cujas amostras revelaram cultura pura de $A$. clavatus, estava sendo utilizado em duas propriedades, onde ocorreram surtos da doença em bovinos. Os sinais clínicos iniciaramse cerca de 2 a 6 dias após a administração do subproduto

\footnotetext{
${ }^{1}$ Recebido em 23 de setembro de 2008.

Aceito para publicação em 6 de outubro de 2008.

Parte da Tese de Doutorado do primeiro autor no Curso de Ciências Veterinárias da Universidade Federal do Rio Grande do Sul (UFRGS).

2 Setor de Patologia Veterinária, Universidade Federal de Lavras (UFLA), Lavras, MG 37.200-000, Brasil.

${ }^{3}$ Setor de Patologia Veterinária, UFRGS, Av. Bento Gonçalves 9090, Agronomia, Porto Alegre, RS 91540-000, Brasil. *Autor para correspondência: davetpat@ufrgs.br

${ }^{4}$ Laboratório de Micologia da Faculdade de Veterinária, UFRGS, Porto Alegre, RS.
}

ou da cultura e a evolução clínica foi de 1,5 a 12 dias. Os sinais clínicos, que foram predominantemente locomotores e respiratórios, incluíram tremores musculares, hiperestesia, taquipnéia progressiva, rigidez de membros pélvicos (mais evidente à locomoção), fraqueza dos posteriores e decúbito. Um ovino também apresentou apoio ocasional sobre os boletos dos membros pélvicos. As anormalidades locomotoras e tremores eram intensificados pelo exercício. Entretanto, em 6 dos 7 ovinos, o apetite e a dipsia eram mantidas até próximo à morte ou eutanásia. O principal achado histológico consistia de degeneração e necrose neuronal cromatolítica em núcleos nervosos específicos do tronco encefálico, cornos ventrais da medula espinhal e gânglios espinhais, trigeminal, estrelado e celíaco. Três ovinos também apresentaram degeneração e necrose leves em músculos dos membros pélvicos e torácicos.

TERMOS DE INDEXAÇÃO: Aspergillus clavatus, ovinos, bagaço de malte, intoxicação, cromatólise neuronal. 


\section{INTRODUÇÃO}

Aspergillus clavatus é um fungo saprófito cosmopolita com importante potencial de risco quando se desenvolve nos cereais e seus subprodutos utlizados na alimentação animal (Varga et al. 2003). O consumo de alimentos contaminados pelo fungo $A$. clavatus tem sido associado com uma doença neurológica em bovinos e ovinos em diversos países, incluindo o Brasil (Kellerman it al. 1976, 1984, Gilmour et al. 1989, Van Der Lugt et al. 2002, Loretti et al. 2003, ElHage \& Lancaster 2004, McKenzie et al. 2004). A natureza da(s) micotoxina(s) envolvida(s) nessa doença ainda não está esclarecida (McKenzie et al. 2004). Várias substâncias têm sido identificadas em culturas e materiais contaminados por A. clavatus, como patulina, brefeldina A, C-sarcina, citocalasina E e K, ascladiol, clavatol, ácido cójico e diversas substâncias tremorgênicas, como triptoquivalona, nortriptoquivalona, triptoquivalina e gliantripina (Demain et al. 1976, Kellerman et al. 1976, 1984, Varga et al. 2003). Embora nenhuma dessas tenha sido testada em ruminantes para determinar sua participação na patogênese da doença (McKenzie et al. 2004), alguns autores especulam que a enfermidade possa resultar da ação sinérgica de várias destas micotoxinas (Varga et al. 2003).

$\mathrm{O}$ isolamento de $A$. clavatus de alimentos contaminados não é suficiente para estabelecer o diagnóstico (Loretti et al. 2003), pois certas cepas de $A$. clavatus podem não ser toxigênicas (Varga et al. 2003). A detecção de micotoxinas reforça o diagnóstico, no entanto, como as toxinas responsáveis pela doença não estão estabelecidas (McKenzie et al. 2004), sua simples presença também não é conclusiva. Reproduções experimentais da neurotoxicose para estabelecer a relação da doença com os alimentos contaminados e o fungo têm sido realizadas em bovinos e ovinos (Kellerman et al. 1976, 1984, Gilmour et al. 1989, Loretti et al. 2003, Colodel et al. 2004). Tais reproduções têm empregado alimentos naturalmente ou experimentalmente contaminados por $A$. clavatus. Exemplos de alimentos associados com doença espontânea e já empregados em experimentos incluem resíduos de destilarias e cervejarias a base de cevada (Gilmour et al. 1989, Loretti et al. 2003) e sorgo (Kellerman et al. 1976) e brotos de milho (Kellerman et al.1984). Este trabalho descreve a toxidez em ovinos do bagaço de malte contaminado com A. clavatus que foi associado com doença e morte de bovinos em duas propriedades no Rio Grande do Sul. A toxidez de isolados do fungo também foi verificada em ovinos. Além disso, os achados clinicos e patológicos da doença induzida nos ovinos são apresentados e discutidos.

\section{MATERIAL E MÉTODOS}

Doses e administrações do bagaço de malte são apresentados no Quadro 1. O bagaço de malte contaminado foi obtido em duas propriedades (Propriedades 1 e 2) onde ocorreram surtos confirmados de neurotoxicose causada por Aspergillus clavatus (Bezerra Jr et al. 2008). Este subproduto foi administrado para três ovinos por via oral com seringa descartável de $60 \mathrm{ml}$ com a extremidade cortada. As administrações foram mantidas até o surgimento de sinais clínicos da enfermidade.

Isolados de $A$. clavatus, obtidos do bagaço de malte das propriedades onde ocorreram os surtos da doença em bovinos (Loretti et al. 2003, Bezerra Jr et al. 2008), foram utilizados para reprodução experimental com cultura fúngica. Estes isolados, armazenados no Laboratório de Micologia da Faculdade de Veterinária, Universidade Federal do Rio Grande do Sul, em água destilada estéril, seguindo a técnica de Castellani (Diogo et al. 2005), foram repicados em Agar Sabouraud e incubados por 10 dias, a temperatura de $25-30^{\circ} \mathrm{C}$. Hifas e conídeos obtidos desse cultivo foram inoculados em meio líquido de Lactrimel ( $14 \mathrm{~g}$ de farinha de trigo, $14 \mathrm{~g}$ de leite em pó, $7 \mathrm{~g}$ de mel e $0,4 \mathrm{~g}$ de cloranfenicol/1l) para obtenção de subcultivos. Imediatamente após inoculação e agitação vigorosa de todos os componentes, o meio líquido inoculado foi distribuído em placas de Petri e incubado por $10-15$ dias, a $25-30^{\circ} \mathrm{C}$, para obtenção de uma densa e uniforme camada de $A$. clavatus. O período e temperatura de incubação utilizados foram estabelecidos em estudos prévios (Demain et al. 1976, Kellerman et al. 1976). Após o período de incubação, foram realizadas novas observações de caracteres macro e micromorfológicos para verificação da pureza do inóculo. Subsequentemente, os meios de cultura com

\begin{tabular}{|c|c|c|c|c|c|c|}
\hline Ovinos & $\begin{array}{l}\text { Dose } \\
\text { diária }\end{array}$ & $\begin{array}{l}\text { Número } \\
\text { de admi- } \\
\text { nistrações }\end{array}$ & Dose total & $\begin{array}{l}\text { Observação de } \\
\text { sinais após } 1^{\text {a }} \\
\text { administração }\end{array}$ & $\begin{array}{l}\text { Evolução } \\
\text { clínica }\end{array}$ & Desfecho \\
\hline $1^{\mathrm{a}}$ & $40 \mathrm{~g} / \mathrm{kg}$ & 3 & $120 \mathrm{~g} / \mathrm{kg}$ & 51 horas & 39 horas & Morte \\
\hline $2^{\mathrm{a}}$ & $40 \mathrm{~g} / \mathrm{kg}$ & 2 & $80 \mathrm{~g} / \mathrm{kg}$ & 51 horas & 67 horas & Morte \\
\hline $3^{b}$ & $12 \mathrm{ml} / \mathrm{kg}$ & $\overline{6}$ & $72 \mathrm{ml} / \mathrm{kg}$ & 72 horas & 72 horas & Eutanásia ${ }^{\mathrm{e}}$ \\
\hline $4^{c}$ & $40 \mathrm{~g} / \mathrm{kg}$ & 19 & $760 \mathrm{~g} / \mathrm{kg}$ & 147 horas & 288 horas & Eutanásia ${ }^{\mathrm{e}}$ \\
\hline $5^{d}$ & $12 \mathrm{ml} / \mathrm{kg}$ & 6 & $72 \mathrm{ml} / \mathrm{kg}$ & 72 horas & 96 horas & Morte \\
\hline $6^{d}$ & $12 \mathrm{ml} / \mathrm{kg}$ & 4 & $48 \mathrm{ml} / \mathrm{kg}$ & 72 horas & 37 horas & Morte \\
\hline $7^{d}$ & $12 \mathrm{ml} / \mathrm{kg}$ & 4 & $48 \mathrm{ml} / \mathrm{kg}$ & 72 horas & 96 horas & Recuperou $^{f}$ \\
\hline
\end{tabular}

a Experimento com bagaço de malte da Propriedade 1, b Experimento com culturas de Aspergillus clavatus em meio lactrimel líquido (Loretti et al. 2003), c Experimento com bagaço de malte da propriedade 2, ${ }^{\mathrm{d}}$ Experimento com culturas de Aspergillus clavatus em meio lactrimel líquido (Bezerra Jr et al. 2008)], e eutanásia com barbitúricos, ${ }^{f}$ eutanásia com barbitúricos dois dias após recuperação clínica. 
o fungo foram homogeneizados em liquidificador doméstico e administrados (12ml/kg até o surgimento de sinais clínicos) por sonda gástrica aos ovinos. Os animais foram mantidos em baias, receberam feno de alfafa e água à vontade, inspecionados duas vezes ao dia e examinados clinicamente (temperatura, freqüência respiratória, cardíaca e ruminal, além da coloração das mucosas externas) uma vez ao dia.

Os ovinos foram necropsiados após morte espontânea ou eutanásia in extremis com barbitúricos. Fragmentos de diversos tecidos foram fixados em formol $10 \%$ tamponado e preparados rotineiramente para histologia. Cortes $(5 \mathrm{~mm})$ foram corados pela hematoxilina e eosina e pelos métodos de violeta de cresil e azul rápido de luxol (Prophet et al. 1992). Amostras do sistema nervoso central incluíram cortes da medula oblonga ao nível do óbex, ponte com pedúnculos cerebelares (ao nível do sulco bulbo-pontino), córtex cerebelar (ao nível do corte anterior), mesencéfalo ao nível dos colículos rostrais, tálamo caudal (secção caudal ao corpo mamilar) e cranial (ao nível do quiasma óptico), córtex cerebral nos lobos occiptal (ao nível do corte mais caudal do tálamo) e frontal (na altura do sulco cruzado), e medula espinhal ao nível torácico e das intumescências lombar e cervical. A classificação dos núcleos lesados seguiu a descrição neuroanatômica de Johnson et al. (2007). Amostras de músculos dos membros pélvicos (semitendinoso, vasto lateral, vasto medial e gastrocnêmio), membros torácicos (tríceps braquial e subescapular), axiais (longíssimo dorsal e psoas), respiratórios (intercostal e diafragma) e cardíacos (ventrículos e septo interventricular) dos animais foram mantidas em geladeira $\left(4^{\circ} \mathrm{C}\right)$ por cerca de 24 horas antes da fixação em formol para evitar artefatos de contração. Foram avaliados ainda fragmentos dos seguintes órgãos: pulmão, fígado, rim, pâncreas, adrenal, tireóide, hipófise, baço, linfonodos, rúmen, reticulo, omaso, abomaso, intestinos e bexiga.

Cortes selecionados do sistema nervoso foram submetidos à imuno-histoquímica através do método da steptavidina-biotina marcada (LSAB). Foram utilizados os seguintes anticorpos primários: a) anti-proteína fibrilar glial ácida (anti-GFAP), b) policlonal de coelho anti-neurofilamento bovino $\mathrm{H}$ de $200 \mathrm{kD}$ (Serotec: AHP245), c) monoclonal anti-proteína prion (VRMD: F99/97).

Para pesquisa de patulina, foram enviadas ao Laboratório de Análises Toxicológicas (Lamic) da Universidade Federal de Santa Maria placas de Petri com a cultura de $A$. clavatus em meio líquido Lactrimel utilizada na reprodução experimental. $\mathrm{A}$ pesquisa de patulina foi realizada por cromatografia líquida de alta eficiência, com detecção por ultravioleta (HPLC/UV).

\section{RESULTADOS}

O tempo até o início dos sinais clínicos, a duração do quadro clínico e o desfecho dos experimentos são apresentados no Quadro 1.

Os sinais clínicos de todos os ovinos foram predominantemente locomotores, os quais, à exceção do Ovino 4, eram acrescidos de sinais respiratórios. Os sinais locomotores de todos ovinos incluíram tremores musculares de intensidade crescente, hiperestesia ao toque e ao som, caminhar com passos curtos, com membros pélvicos afastados, rígidos e dando pequenos saltos. Os ovinos ficavam sempre atentos ao ambiente com orelhas eretas. Inicialmente os tremores e a hiperestesia eram pouco perceptíveis, especialmente quando os animais estavam em repouso. A movimentação es- pontânea ou forçada tornava esses sinais mais intensos e, com a progressão da doença, ocasionalmente levava ao decúbito. A manutenção da cabeça do animal erguida, por cerca de um minuto, também causava efeito semelhante, quando esta era solta subitamente [Teste HR ou "head raising test"]. Após períodos variáveis de repouso, os animais ficavam novamente em estação, mas com dificuldade que aumentava progressivamente. Nas fases finais, os animais apresentavam grande dificuldade de manter a estação e permaneciam a maior parte do tempo em decúbito esternal. Quando em estação, os membros pélvicos eram deslocados cranialmente e freqüentemente afastados; os membros torácicos eram aproximados, deslocados caudalmente e trêmulos; enquanto a cabeça permanecia abaixo da linha do corpo. Os animais demonstravam progressiva fraqueza muscular nos membros pélvicos, deslocando o apoio para os membros torácicos (Fig.1). O Ovino 4, nesta fase da doença ocasionalmente apoiava-se sobre os boletos dos membros pélvicos.

Exceto pelo Ovino 4, nos demais, a freqüência respiratória aumentou progressivamente e evoluiu para respiração com narinas dilatadas e, posteriormente, ofegante com a boca aberta e a língua exposta (Fig.2). O apetite permaneceu nesses ovinos até próximo da morte ou eu-

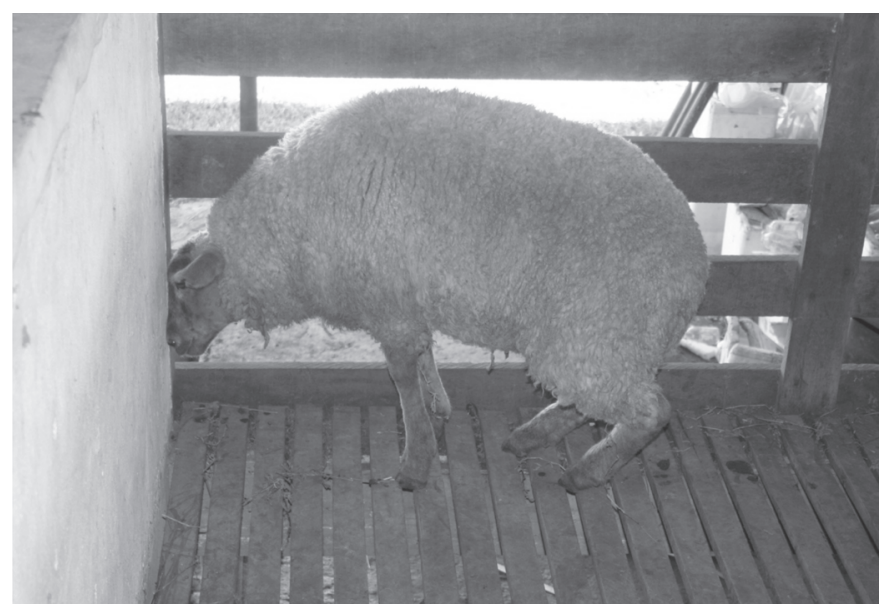

Fig.1. Neurotoxicose causada por Aspergillus clavatus em ovinos (Ovino 2). Animal demonstra fraqueza nos membros pélvicos e deslocamento do apoio para os membros torácicos.

tanásia. O Ovino 4, entretanto, apresentou anorexia e apatia com a progressão da doença clínica. A temperatura corporal do Ovino 3 ultrapassou os $42^{\circ} \mathrm{C}$ próximo da morte. Os movimentos ruminais, a freqüência cardíaca e o tônus da língua dos animais não apresentaram alterações significativas durante o período de experimento.

Não houve alterações significativas nas necropsias. $\mathrm{Na}$ histologia, as principais alterações foram observadas no sistema nervoso e músculos esqueléticos. Os achados microscópicos no sistema nervoso se caracterizavam por lesões degenerativas e necróticas, cujas respectivas áreas anatômicas e intensidades estão apresentadas no Quadro 2. As lesões eram bilaterais e relativamente si- 


\section{Quadro 2. Intoxicação experimental por Aspergillus clavatus em ovinos. Áreas e estruturas do sistema nervoso com lesões microscópicas e suas respectivas intensidades $^{a}$}

\begin{tabular}{|c|c|c|c|c|c|c|c|}
\hline $\begin{array}{l}\text { Áreas ou estruturas do } \\
\text { sistema nervoso com lesões }\end{array}$ & $\begin{array}{c}\text { Ovino } \\
1\end{array}$ & $\begin{array}{l}\text { Ovino } \\
2\end{array}$ & $\begin{array}{l}\text { Ovino } \\
3\end{array}$ & $\begin{array}{l}\text { Ovino } \\
4\end{array}$ & $\begin{array}{l}\text { Ovino } \\
5\end{array}$ & $\begin{array}{l}\text { Ovinc } \\
6\end{array}$ & $\begin{array}{l}\text { Ovin } \\
7\end{array}$ \\
\hline \multicolumn{8}{|l|}{ Intumescência lombar } \\
\hline Corno ventral & + & + & + & + & ++ & + & ++ \\
\hline Corno dorsal & 0 & 0 & 0 & 0 & 0 & 0 & \\
\hline \multicolumn{8}{|l|}{ Intumescência cervical } \\
\hline Corno ventral & 0 & + & + & 0 & + & 0 & + \\
\hline Corno dorsal & 0 & 0 & 0 & 0 & 0 & 0 & D \\
\hline \multicolumn{8}{|l|}{ Medula torácica } \\
\hline Corno ventral & + & + & + & 0 & + & + & + \\
\hline Corno dorsal & 0 & 0 & 0 & 0 & 0 & 0 & \\
\hline \multicolumn{8}{|l|}{ Bulbo ao nível do óbex } \\
\hline $\begin{array}{l}\text { Núcleo motor dorsal } \\
\text { do nervo vago }\end{array}$ & +++ & +++ & +++ & + & +++ & +++ & 0 \\
\hline Núcleo ambíguo & 0 & + & ++ & 0 & + & + & 0 \\
\hline \multicolumn{8}{|l|}{ Cerebelo / Ponte } \\
\hline Núcleos vestibulares & + & 0 & + & + & ++ & + & ++ \\
\hline $\begin{array}{l}\text { Formação reticular da ponte } \\
\text { Tálamo caudal ao corpo ma- } \\
\text { milar / colículos rostrais }\end{array}$ & + & + & + & ++ & +++ & + & ++ \\
\hline Núcleo vermelho & ++ & +++ & +++ & + & +++ & ++ & + \\
\hline Gânglio espinhal & ++ & +++ & +++ & ++ & +++ & - & - \\
\hline Gânglio trigeminal & + & +++ & +++ & +++ & + & +++ & +++ \\
\hline Gânglio estrelado & - & - & ++ & - & ++ & + & 0 \\
\hline Gânglio celíaco & - & ++ & + & + & + & + & 0 \\
\hline Nervo isquiádico & 0 & 0 & 0 & 0 & - & 0 & \\
\hline Nervo fibular & - & - & 0 & 0 & 0 & 0 & \\
\hline Plexo braquial & - & - & 0 & 0 & 0 & - & \\
\hline Nervo vago & - & - & 0 & 0 & 0 & 0 & 0 \\
\hline
\end{tabular}

a Intensidade das lesões: (+) leve (1-5 neurônios cromatolíticos), (++) moderada (6-10 neurônios cromatolíticos), $(+++)$ acentuada (mais que 10 neurônios cromatolíticos), (0) sem alterações, (-) não pesquisado, nos nervos as cruzes se referem à intensidade das lesões axonais.

métricas. Não foram observadas lesões nos plexos mioentéricos e submucosais nos cortes de intestino dos animais deste estudo.

Os neurônios afetados apresentavam tumefação, citoplasma eosinofílico, homogêneo e pálido, particularmente na porção central do pericário. Os grânulos de Nissl estavam ausentes ou rarefeitos e dispostos na periferia da célula (cromatólise central) (Fig.3 e 4), porém alguns neurônios apresentavam a rarefação na periferia do pericário (cromatólise periférica). Os núcleos dos neurônios afetados eram, com freqüência, periféricos e, ocasionalmente, com picnose, cariorrexia ou cariólise. Outros neurônios se apresentavam retraídos e com evidente eosinofilia citoplasmática, além de picnose ou cariólise.

A tumefação neuronal e as alterações nucleares regressivas foram variáveis entre os ovinos deste estudo e também entre áreas afetadas. Nos Ovinos 4, 5 e 7, as intensidades destas variaram de leves a acentuadas, sendo mais intensas nos núcleos vestibulares laterais, formação reticular da ponte (Ovinos 4, 5 e 7) e vermelhos (Ovino 5). Nestes três ovinos o óbito ocorreu de 96 a 288 horas após o início dos sinais clínicos. Estas alterações eram leves nos demais ovinos de evolução clínica relativamente mais curta (37-72 horas).

A imunomarcação do neurofilamento foi observada

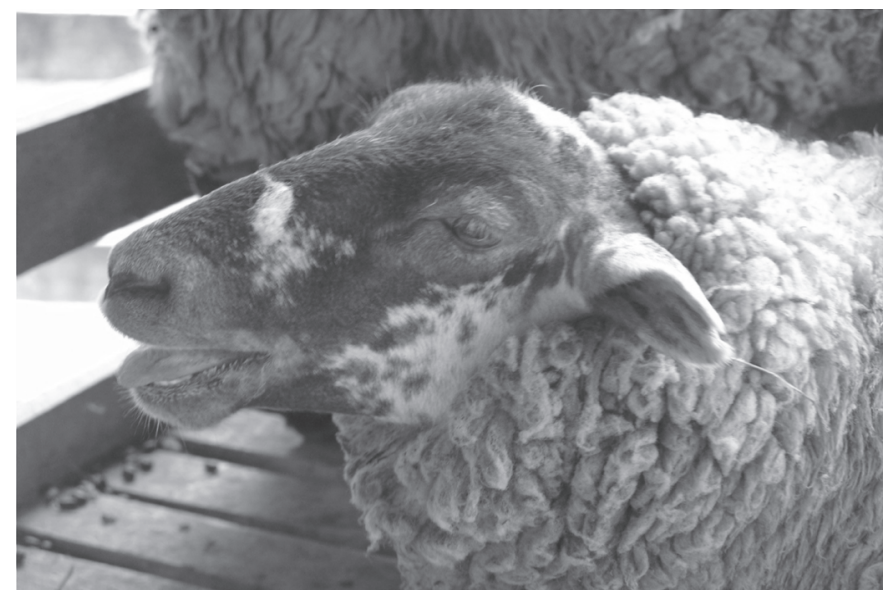

Fig.2. Neurotoxicose causada por Aspergillus clavatus em ovinos (Ovino 3). Animal respira com a boca aberta e expondo a língua.

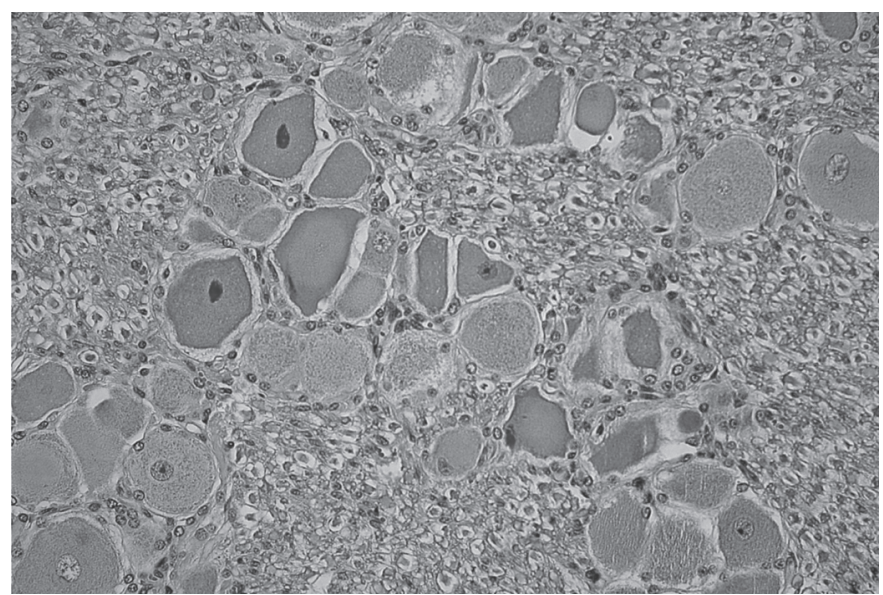

Fig.3. Neurotoxicose causada pelo Aspergillus clavatus em ovinos (Ovino 3). Gânglio trigêmio. Neurônios com rarefação ou ausência dos grânulos de Nissl (cromatólise) e núcleos picnóticos centrais ou periféricos. HE, obj.20x.

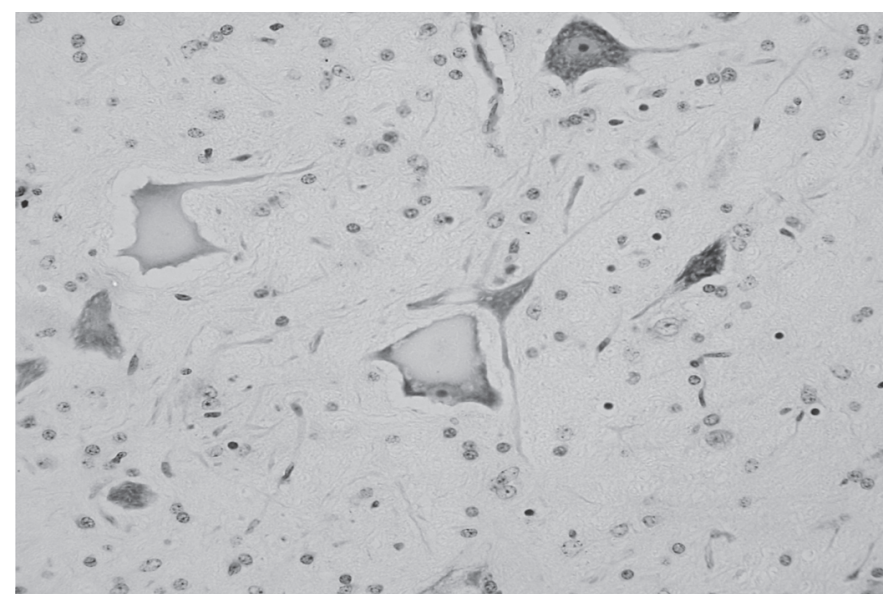

Fig.4. Neurotoxicose causada pelo Aspergillus clavatus em ovinos (Ovino 7). Núcleo vermelho. Neurônios com cromatólise central e núcleos periféricos ou ausentes. Observe neurônios sem alterações, com citoplasma contendo granulação azulada abundante, adjacentes aos neurônios afetados. Violeta de Cresil, obj.40x. 
apenas em axônios do sistema nervoso central e nervos periféricos, não sendo notada marcação no pericário de neurônios cromatolíticos. Não foi evidenciado aumento na imunomarcação da GFAP em astrócitos das áreas contendo neurônios cromatolíticos. A imunoistoquímica para PrP nestes animais foi consistentemente negativa.

Havia necrose hialina e flocular, segmentar e aguda nos músculos semitendinoso (Ovino 3), vasto lateral (Ovino 3), vasto medial (Ovinos 1 e 3), gastrocnêmio (Ovinos 3 e 4), tríceps braquial (Ovino 4) e subescapular (Ovinos 1,3 e 4). Estas lesões foram leves em quase todos os animais afetados, com exceção do Ovino 3, no qual eram moderadas. Os demais ovinos não apresentaram lesões em nenhum dos músculos avaliados.

\section{DISCUSSÃO}

O bagaço de malte proveniente dos surtos da doença em bovinos bem como os isolados de Aspergillus clavatus foram tóxicos para ovinos. Os achados clínicos e patológicos desses ovinos foram semelhantes aos descritos em bovinos e ovinos na doença neurológica espontânea associada com ingestão de alimentos contaminados por $A$. clavatus e reproduzida experimentalmente (Kellerman et al. 1976, 1984, Gilmour et al. 1989, Shlosberg et al. 1991, Van der Lugt et al. 2002, Loretti et al. 2003, Colodel et al. 2004, El-Hage \& Lancaster 2004, McKenzie et al. 2004, Bezerra Jr et al. 2008). Os experimentos com os isolados de $A$. clavatus do bagaço de malte das propriedades evidenciam envolvimento do fungo nos surtos de bovinos. Esta metodologia pode ser útil para comprovação do envolvimento do fungo em surtos futuros.

O início e a duração dos sinais clínicos observados nesse estudo foram semelhantes aos descritos na literatura (Kellerman et al. 1976, 1984). Entretanto, o Ovino 4, que recebeu bagaço de malte da Propriedade 2, apresentou maior tempo para o início dos sinais clínicos e maior duração do quadro clínico. Estes dados sugerem que o subproduto desta propriedade era menos tóxico em relação ao da Propriedade 1. Por outro lado, a semelhança nos tempos de início dos sinais clínicos e evolução clínica sugerem que a toxidez do subproduto da Propriedade 1 foi comparável à da cultura pura do fungo. Essa aparente superior toxicidade do subproduto da Propriedade 1 talvez possa estar associada com a maior letalidade observada naquele surto (Bezerra Jr et al. 2008).

Uma vez que $A$. clavatus pode produzir várias micotoxinas tremorgências e causar lesões em neurônios do tronco encefálico e medula espinhal (Kellerman et al. 1976, 1984, Varga et al. 2003), os sinais clínicos locomotores podem estar relacionados à ação destas micotoxinas e/ ou deficiência na função de neurônios afetados. Foram descritas lesões histológicas em ovinos intoxicados que não demonstraram sinais clínicos (Gilmour et al. 1989, Loretti et al. 2003). Fato semelhante pode ser observado no Ovino 7 que se recuperou, o que sugere que tanto a intensidade das lesões, quanto a ação das micotoxinas tremorgênicas sejam importantes para manifestação clínica da doença causada por $A$. clavatus.
A hipertermia, observada no Ovino 3 , é descrita apenas raramente na neurotoxicose causada por $A$. clavatus em bovinos (Kellerman et al. 1976) e pode ter sido associada à constante estimulação muscular pelos tremores, como ocorre em estados convulsivos (De Lahunta 1983). A taquipnéia e respiração ofegante, observada em 6 de 7 ovinos deste estudo, é também descrita por outros autores (Gilmour et al. 1989, Kellerman et al. 1976). No entanto, não foi possível estabelecer uma correlação segura entre as áreas com lesões e a taquipnéia. Lesões na porção medial da ponte têm sido associadas com taquipnéia em humanos (Tarulli et al. 2005). Como os ovinos não apresentavam lesões significativas nos pulmões e apresentavam lesões em neurônios nestas áreas (formação reticular da ponte), a origem neurogênica da taquipnéia não deve ser desconsiderada.

A distribuição da degeneração e necrose neuronal cromatolítica observada nesses casos foi semelhante à descrita previamente (Kellerman et al. 1976, 1984, Gilmour et al. 1989, Shlosberg et al. 1991, Van der Lugt et al. 2002, Loretti et al. 2003, El-Hage \& Lancaster 2004, McKenzie et al. 2004, Colodel et al. 2004, Bezerra Jr. et al. 2008). Os neurônios envolvidos eram neurônios motores superiores e inferiores, sensórios e de gânglios autônomos, denotando uma doença neuronal degenerativa multissistêmica.

Conforme anteriormente descrito, não foram observadas lesões em axônios da medula espinhal (Loretti et al. 2003, McKenzie et al. 2004) ou de nervos periféricos (ElHage \& Lancaster 2004). Sabe-se que, ao menos em algumas neuropatias tóxicas, o surgimento de alterações no pericário pode ocorrer desde as fases iniciais da doença, quando os axônios estão praticamente intactos (Sterman 1983). Tal fato talvez possa explicar a ausência de lesões axonais nos ovinos desse estudo. Lesões axonais provavelmente sejam observadas com maior freqüência em casos mais crônicos na doença causada por $A$. clavatus (Van Der Lugt et al. 2002).

O padrão de distribuição de neurônios afetados observado aqui foi relativamente constante e reproduzível, indicando que os neurônios afetados compartilham características que os tornam sensíveis a agressões específicas (Kellerman et al. 1984). As principais características morfológicas que distinguem a maioria dos neurônios afetados de outros não afetados são seus axônios longos e de maior diâmetro (De Lahunta 1983, Zachary 2007). Por exemplo, em relação aos neurônios motores superiores, os núcleos vermelhos (Chiocchetti et al. 2006), vestibulares laterais (Grandis et al. 2007) e da formação reticular da ponte (De Lahunta 1983) afetados nos ovinos deste estudo, são os principais a emitir projeções axonais ao nível da medula lombar em ruminantes. Outros neurônios motores superiores, como os do córtex cerebral motor, que não apresentam essa característica em ruminantes (De Lahunta 1983), não apresentavam alterações nesses ovinos. Assim, os dados desse trabalho indicam que o comprimento dos axônios seja uma característica importante na susceptibilidade dos neurônios na doença causada por A. clavatus. 
Conforme observado por Kellerman et al. (1976), a pesquisa de patulina na cultura utilizada na reprodução experimental no Ovino 3 foi negativa. Além disso, a administração de patulina em camundongos, embora cause sinais clínicos considerados semelhantes, causa lesões restritas ao córtex cerebral (Capitaine \& Bolouet 1974). Da mesma forma como descrito anteriormente (Loretti et al. 2003), não foram observadas lesões nas áreas de córtex cerebral avaliadas. Assim, a participação desta e de outras micotoxinas no quadro clínicopatológico associado a $A$. clavatus ainda necessita de comprovação (McKenzie et al. 2004). As lesões musculares observadas nesses ovinos e em casos prévios (Kellerman et al. 1976, Gilmour et al. 1989, Loretti et al. 2003) foram leves ou ausentes. A patogênese destas lesões musculares ainda é indeterminada (Gilmour et al. 1989, Loretti et al. 2003, El-Hage \& Lancaster 2004).

Considerando a apresentação clínica da doença causada por $A$. clavatus nos ovinos, o diagnóstico diferencial deve incluir principalmente as síndromes tremorgênicas causadas por Claviceps paspali, Stenocarpella (Diplodia) maydis, Cynodon dactylon, Lolium perenne, Ipomoea asarifolia e Phalaris spp., bem como as doenças de armazenamento lisossomal causadas pelo consumo de Sida carpinifolia, Solanum fastigiatum var. fastigiatum e Ipomoea carnea subsp. fistulosa. Devido a alguns ovinos apresentarem lesões musculares na histologia, o diagnóstico diferencial deve também incluir as miopatias tóxicas e nutricionais (Loretti et al. 2003, Barros et al. 2006). Finalmente, as encefalopatias espongiformes também são importantes diferenciais devido às semelhanças clínicas e histológicas, situações em que a imuno-histoquímica é crucial para diferenciação inequívoca (Loretti et al. 2003, Barros et al. 2006). Nos casos aqui apresentados a imunohistoquímica em cortes da medula oblonga ao nível do óbex de todos ovinos foram consistentemente negativas.

Agradecimentos.- Este estudo teve suporte financeiro do Conselho Nacional de Desenvolvimento Científico e Tecnológico (CNPq) e da Coordenação de Aperfeiçoamento de Pessoal de Nível Superior (CAPES). Os autores agradecem ao Professor Cláudio Estevão Farias da Cruz (UFRGS) pela revisão deste artigo e aos Professores Rodolfo Voll (UFRGS) e Marcos Dutra Duarte (UFPA) pelo auxílio na interpretação dos sinais clínicos.

\section{REFERÊNCIAS}

Barros C.S.L., Driemeier D., Dutra I.S. \& Lemos R.A.A. 2006. Doenças do Sistema Nervoso de Bovinos no Brasil. AGNS Gráfica e Editora, São Paulo. 207p.

Bezerra Jr P.S., Raymundo D.L., Spanamberg A., Corrêa A.M., Bangel Jr J.J., Ferreiro L. \& Driemeier D. 2008. Neurotoxicose em bovinos associada ao consumo de bagaço de malte contaminado por Aspergillus clavatus. Pesq. Vet. Bras. 28(3):210-218.

Capitaine R. \& Balouet G. 1974. Étude histopathologique des lésions induites chez la souris par des injections intra-peritoneales et intracérébrales de patuline. Mycopathol. Mycol. Appl. 54(3):361-368.

Chiocchetti R., Bombardi C., Grandis A., Mazzuoli G., Gentile A., Pisoni L., Joechler M. \& Lucchi M.L. 2006. Cytoarchiteture, morphology, and lumbosacral spinal cord projections of the red nucleus in cattle. Am. J. Vet. Res. 67(10):1662-1669.
Colodel E.M., Schmitz M., Traverso S.D., Sanches E.M.C., Ferreiro F., Loretti A.P., Correa A.M.C., Seitz A.L. \& Driemeier D. 2004. Aspectos clínicos e patológicos da doença neurológica de bovinos reproduzida pela administração de milho contaminado com Aspergillus clavatus. Pesq. Vet. Bras. 24(Supl.):16-17.

De Lahunta A. 1983. Veterinary Neuroanatomy and Clinical Neurology. $2^{\text {nd }}$ ed. W.B. Saunders, London. 471p.

Demain A.L., Hunt N.A., Malik V., Kobbe B., Hawkins H., Matsuo K. \& Wogan G.N. 1976. Improved procedure for production of cytochalasin $\mathrm{E}$ and tremorgenic mycotoxins by Aspergillus clavatus. Appl. Environ. Microbiol. 31(1):138-140.

Diogo H.C., Sarpieri A. \& Pires M.C. 2005. Preservação de fungos em água destilada. An. Bras. Dermatol. 80(6):591-594.

El-Hage C.M. \& Lancaster M.J. 2004. Mycotoxic nervous disease in cattle fed sprouted barley contaminated with Aspergillus clavatus. Aust. Vet. J. 82(10):639-641.

Gilmour J.S., Inglis D.M., Robb J. \& Maclean M.A. 1989. A fooder mycotoxicosis of ruminants caused by contamination of distillery byproduct with Aspergillus clavatus. Vet. Rec. 124(6):133-135.

Grandis A., Bombardi C., Travostini B., Gentile A., Joechler M., Pisoni L. \& Chiocchetti R. 2007. Vestibular nuclear complex in cattle: Topography, morphology, cytoarchitecture and lumbo-sacral projections. J. Vestib. Res. 17(1):9-24.

Johnson J.I., Sudheimer K.D., Davis K.K., Kerndt G.M. \& Winn B.M. 2007. The Sheep Brain Atlas. https://www.msu.edu/ brains/brains/ sheep/index.html. Consultado em 12 março de 2007.

Kellerman T.S., Pienaar J.G., Van der Westhuizen G.C.A., Anderson L.A.P. \& Naudé T.W. 1976. A highly fatal tremorgenic mycotoxicosis of cattle caused by Aspergillus clavatus. Onderstepoort J. Vet. Res. 43(3):147-154.

Kellerman T.S., Newsholme S.J., Coetzer J.A.W. \& Van der Westhuizen G.C.A. 1984. A tremorgenic mycotoxicosis of cattle caused by maize sprouts infested with Aspergillus clavatus. Onderstepoort J. Vet. Res. 51(4):271-274.

Loretti A.P., Colodel E.M., Driemeier D., Corrêa A., Bangel Jr J.J. \& Ferreiro L. 2003. Neurological disorder in dairy cattle associated with consumption of beer residues contaminated with Aspergillus clavatus. J. Vet. Diagn. Invest. 15(2):123-132.

McKenzie R.A., Kelly M.A., Shivas R.G., Gibson J.A., Cook P.J., Widderick K. \& Guilfoyle A.F. 2004. Aspergillus clavatus tremorgenic neurotoxicosis in cattle fed sprouted grains. Aust. Vet. J. 82(10):635-638.

Prophet E.B., Mills B., Arrington J.B., Leslie H. \& Sobin L.H.M.D. 1995. AFIP Laboratory Methods in Histotechnology. The Registry of Pathology, Washington, DC. 280p.

Shlosberg A., Zadikov I., Perl S., Yakobson B., Varod Y., Elad D., Rapoport E. \& Handji V. 1991. Aspergillus clavatus as the probable cause of a lethal mass neurotoxicosis in sheep. Mycopathologia. 114(1):35-39.

Sterman A.B. 1983. Altered sensory ganglia in acrilamide neuropathy: quantitative evidence of neuronal reorganization. J. Neuropathol. Exp. Neurol. 42(2):166-176.

Tarulli A.W., Lim C., Bui J.D., Saper C.B. \& Alexander M.P. 2005. Central neurogenic hyperventilation: A case report and discussion of pathophysiology. Arch. Neurol. 62(10):1632-1634.

Van der Lugt J.J., Prozesky L. \& Van Wilpe E. 2002. Acute Aspergillus clavatus poisoning in cattle: Light microscopical and ultrastructural lesions in the spinal cord, p.131-141. In: Van der Lugt J.J. (Ed.), The Clinicopathology and Pathology of Selective Toxicoses and Storage Diseases of the Nervous System of Ruminants in Southern Africa. Doctoral Thesis, University of Utrecht, Netherlands.

Varga J., Rigó K., Molnár J., Tóth B., Szencz S., Téren J. \& Kozakiewicz Z. 2003. Mycotoxin production and evolutionary relationships among species of Aspergillus section Clavati. Antonie van Leeuwenhoek 83(2):191-200.

Zachary J.F. 2007. Nervous system, p.833-972. In: McGavin M.D. \& Zachary J.F. (Ed.), Pathologic Basis of Veterinary Disease. $4^{\text {th }}$ ed. Mosby Elsevier, St Louis, Mo. 